\title{
Methodology of Intelligent Energy Management System Simulation for Electric Vehicle Applications with Asynchronous Logic Controller
}

\author{
Ilya Kavalchuk ${ }^{1,2}$, Huy Le Nguyen ${ }^{1,2}$, Thanh Pham $^{1,2}$, Alex Stojcevski ${ }^{1}$ \\ ${ }^{1}$ Centre of Technology \\ RMIT University Vietnam \\ Ho Chi Minh City, Vietnam \\ ${ }^{2}$ School of Electrical and Computer Engineering \\ RMIT University \\ Melbourne, Australia \\ e-mail: ilya.kavalchuk@rmit.edu.vn
}

\begin{abstract}
Power management in electrical vehicles has become significantly a challenging task due to the growing number of sub-systems, components and energy storage systems development. Moreover, efficient modelling, simulation, evaluation and optimization of power consumption is further complicated due to the asynchronous nature of power supply requests from those sources. This paper describes a methodology of modeling and simulation of an intelligent energy management system for electric vehicles in a MatLab Simulink environment. A centralized control architecture with an asynchronous-based controller was proposed as a novel design and implementation approach in order to optimize the computational time and energy consumption for real time applications with stochastic load requests in line with balancing the load request between the components of the hybrid energy storage system.
\end{abstract}

Keywords - asynchronous logic, control of intelligent systems, electric vehicles, smart energy management system

\section{INTRODUCTION}

Simulation of the energy consumption in complex electrical system requires a deep understanding of the principals of energy consumption, the sources of the energy requests and the nature of the power suppliers. Electric vehicles (EVs) are a good example of such systems with a variety of different devices integrated in the system, along with various voltage busses and bidirectional powerflow [1].

The main power consumers in an electric vehicle are the powertrain and the climate control system (HVAC). But modern vehicles can be considered as more complicated systems, which include a large number of the sub-systems and components [2]. At the same time, the available supplies to all the energy requests are limited to the capacity of the energy storage systems (ESS). The most common energy storage system for EV application is a battery pack. Modern research shows that there are several problems aligned with the usage of the battery pack as an individual power source for EV. To overcome these major problems, hybrid ESS is being developed in order to increase the efficiency of the ESS for all operational modes [3].

Another major problem for reliable energy consumption simulation for EVs is the nature of the power requests. The usage of systems as the result of the requests coming from several sources. The first source of request is the occupants inside the vehicle. People may turn on or off systems to create a comfortable and safe environment for themselves. The second request sources are the sensors of various systems which operate independently. The best example of such systems is the active safety systems, such as ABS (Anti-lock Braking System). [4]

Both request sources have stochastic and unpredictable nature, which makes the simulation process more complicated. For drivetrain analysis, one of the predeveloped driving cycles can be used in order to achieve repeatability of the experiments and smoothen the transition from the simulation environment to real world application. [5] At the same time, the power consumption of the additional systems may be higher than the single power consumption of the drivetrain and their consumption cannot be predefined which makes the simulation process very complicated.

Asynchronous logic design paradigm and its applications have become potentially beneficial than the synchronous counterparts in recent years. Asynchronous architectures provide promising solutions for existing issues inherently associated with contemporary synchronous designs, for instance significant increase in power dissipation, challenges in achieving high speed, high performance and low noise applications [6]. Due to the nature of the asynchronous logic, researchers find it suitable for implementing heterogeneous systems such as modern EVs. Such systems are integrated with multiple digital and analog function blocks that are powered with different power supply domains and are communicating with each other constantly. The variations in system components will likely lead to the challenge in maintaining seamless communications between these components over time. For systems where transmitting signals are non-uniform and discontinuous, asynchronous communication methods are also preferred over clock- 
based ones due to the fact that there is less clock switching activities as well as complicated interrupt algorithms for the synchronous controller. Instead, handshaking communication as shown in Fig. 1 can be adapted where individual requests will be acknowledged and processed.

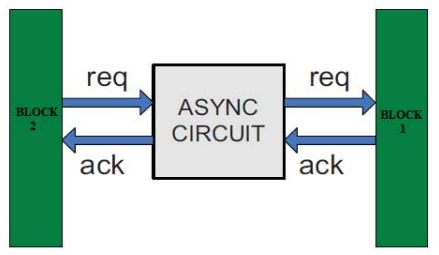

Figure 1. Handshaking communication

There has been a growing interest in adapting asynchronous logic design approaches into power control and management applications due to the reasons discussed earlier. An example is the realisation of asynchronous design methodology in designing smart controllers for the power management unit of wireless sensor nodes. [7] The results suggested that the asynchronous logic benefit is a huge reduction in terms of dynamic energy dissipation along with a significantly reduced functional complexity. Wireless sensor systems are similar to EV system in the sense that both systems are working with unpredictable and random external responses. Signals captured from sensor nodes are often discontinuous and non-uniform, which behaved similarly to signals found in modern EVs in terms of power requests. The functional block from the study above, event detector, was built to work with different start-up conditions, which proposes that asynchronous controllers can be developed to communicate with components in both the ESS and loads [7]. In short, these encourage the adaption of asynchronous logic methodology into developing a centralised controller for an EV system in order to tackle the challenge of unpredictability and compatibility between system components as well as the hybridization of the power sources.

This paper describes the methodology to model and simulate the power consumption of the electric vehicles with a centralized energy management controller designed using asynchronous logic. Two parts of this paper present the application of asynchronous logic to the EV systems as applied to the ESS management as well as the load control applications. A specific asynchronous logic design paradigm - Null Convention Logic (NCL) will be discussed to demonstrate how the logic can be adopted in the design and implementation of the energy management system controllers. The sections present the physical definition of the controlling systems and ESS, application of the asynchronous logic to control the power in the ESS and for the load sharing operations and the simulation of the discussed application in the MatLab Simulink environment.

\section{SYSTEM ARCHITECTURE}

\section{A. Energy Storage System}

ESS is a primary part of the EV as it defines the available energy and usability of the vehicles through its capacity. The most common way to design ESS is to install batteries with the highest capacity in order to fulfill all possible energy-consuming requests from on-vehicle applications and provide reasonable performance in terms of the driving range on a single charge. The major problem with big battery packs is their excessive weight and very long recharging times. Batteries also have limited capability in terms of the charging power, which limits the energy outcome of the recuperative braking process. Efficiency of the recuperative braking processes have a direct impact on the overall efficiency of the drivetrain, as this process is the only way to restore used energy from the ESS in an EV.

To overcome the limitations of batteries during the recuperative braking processes, high power ultracapacitors (UC) can be used, as the capacitors can be recharged with higher power in order to store higher amount of energy and decrease the possible damage to the batteries.

In terms of the size and recharging time characteristics, a fuel cell (FC) is the best option to support the battery pack. The low refueling time combined with relatively high energy density makes fuel cells a good long-term energy storage solution for EVs.

The stand-alone components, ultra-capacitor and fuel cell, cannot completely replace the battery pack even though the battery pack itself has many limitations. UCs have a high power density and they are very efficient for the short-term high-power flow regimes, but the energy density is low, so it is not able to supply the energy for long term operations. Alternatively, FCs have a slower power response in comparison with other ESS. The main power output regulation in FC can be achieved only through the hydrogen flow regulation. As the process of the flow regulation is very slow, the power response of the FC is slow. Furthermore, FC cannot be used to store energy back in the recuperative braking process. Therefore, the best option to fulfill all requirements is to combine various components in a hybrid ESS. A typical hybrid ESS model is represented on Fig. 2.

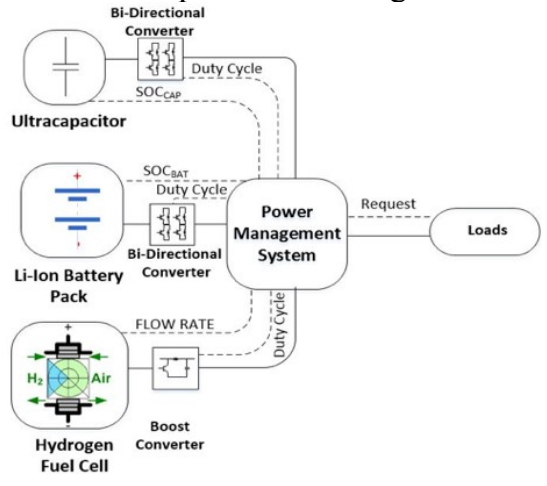

Figure 2. ESS Management System Design 
Solid lines on Fig. 2 represent the power flow, and the dashed lines illustrate the information connections.

In the proposed ESS management system design, the main controlling parameters are the duty cycles of the individual converters to control the power distribution between the battery, $\mathrm{UC}$ and $\mathrm{FC}$. The $\mathrm{FC}$ requires additional control over the hydrogen flow rate in order to control the output power capability and to react to the changes in the status of the loads and other components of ESS. The duty cycle parameters and flow rate regulation are made by the power requests from the load controller. The request from the loads is the main parameter to regulate the energy distribution in the ESS, but the final decision is made based on the States of Charge (SoC) of the UC and battery pack and the hydrogen flow rate of the FC.

Power flow inside the ESS has a more complicated nature. As the battery and UC can be recharged, the converters are bidirectional in nature. This allows the power management system to select the most efficient mechanism to charge the selected elements and to store the maximum amount of the regenerated energy. Bidirectional converters also allow the FC to charge the battery bank when the power consumption is low, the SoC of the battery $\left(\mathrm{SoC}_{\mathrm{BAT}}\right)$ is below the programmed values ( SoC $\left._{\text {CRITICAL }}\right)$ and the hydrogen flow rate is high.

Both the capacitors as well as FC can recharge the battery when $\mathrm{SoC}_{\mathrm{BAT}}$ is low and capacitor is charged. Due to the nature of the energy stored in capacitor, the FC is not allowed to charge the capacitor in any circumstances, as the higher SoC of the capacitor leads to the lower power capability during the regenerative braking process. Furthermore, recuperative braking power can be split between batteries and capacitors based on the SoC of each component, required braking torque and provided current.

The overall ESS power output can be presented as the system of three equations for each component, as shown in (1):

$$
\left\{\begin{array}{c}
P_{F C}=\left(\frac{2 F Q_{H_{2}} P_{H_{2}}}{A_{m} R T}\right)^{2} R_{L} A_{m}=c\left(Q_{H_{2}} P_{H_{2}}\right)^{2} \\
P_{b a t}=f\left(S o C_{b a t}\right) \\
P_{c a p}=f\left(S o C_{c a p}\right)
\end{array}\right.
$$

The general pattern of the decision-making algorithm for the ESS Power Management System Controller is presented on Fig. 3. The main parameters are Required Power $\left(\mathrm{P}_{\text {req }}\right)$, State of Charges for battery and capacitors $\left(\mathrm{SoC} \mathrm{C}_{\text {bat }}\right.$ and $\left.\mathrm{SoC}_{\mathrm{cap}}\right)$ and the hydrogen flow rate for the fuel cell regulation. The available power is specified by the SoC and flow rate using (1).

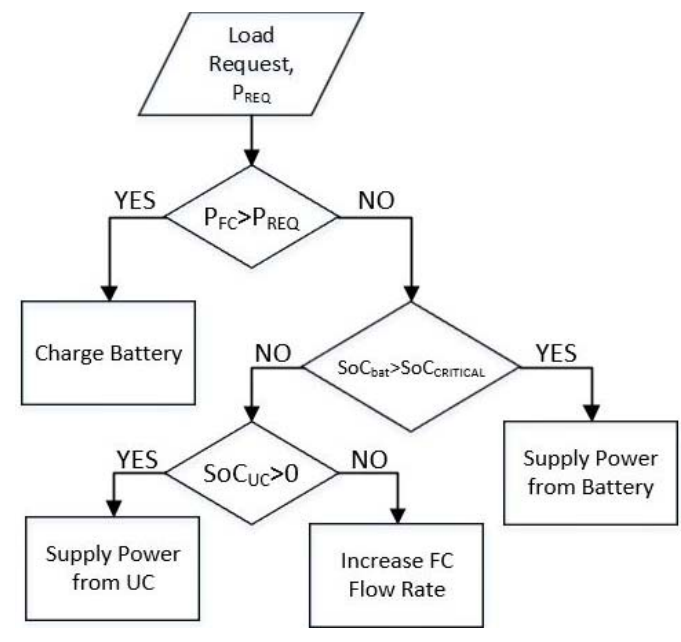

Figure 3. ESS Decision Making Process

\section{B. Loads.}

The loads in an EV are primarily electric in nature due to the unavailability of other sources of energy, such as rotational or thermal energy. Due to the standardization of the components, the electric architecture consists of two relatively separate electrical bus lines. First bus operates on the high voltage, up to $1200 \mathrm{VDC}$, and it is used to supply energy to the components with high power requirements. The powertrain is the main component powered by the high voltage bus line. The energy in this bus line is bidirectional, as the motor is not only consuming, but also supplying the energy back to the ESS. For reliability purposes, the HVAC system is also obtaining power from the high voltage line due to the high power requirements of the components of the HVAC.

The low voltage line is used to supply power to the systems with a low energy requirement. These systems can be easily transferred from the traditional vehicles with minimal changes. While the peak power of the low voltage bus components are lower than that of the high voltage bus components, the operational time of these components have a direct impact on the overall consumed energy and can be higher for some systems and components. This leads to a higher energy consumption by the low power devices and can be comparable with the energy consumption of the systems with a higher peak power and low operational time.

The proposed ESS management system design includes a centralized controller. A centralized system provides possibility to react faster in response and improve the energy demand regulation and connectivity between components in comparison with a decentralized architecture. The main problem of the centralize system is the required computational power, especially, when the controller operates on the conventional clock-dependent logic with the decision making process. The developed system architecture is presented on Fig. 4. 


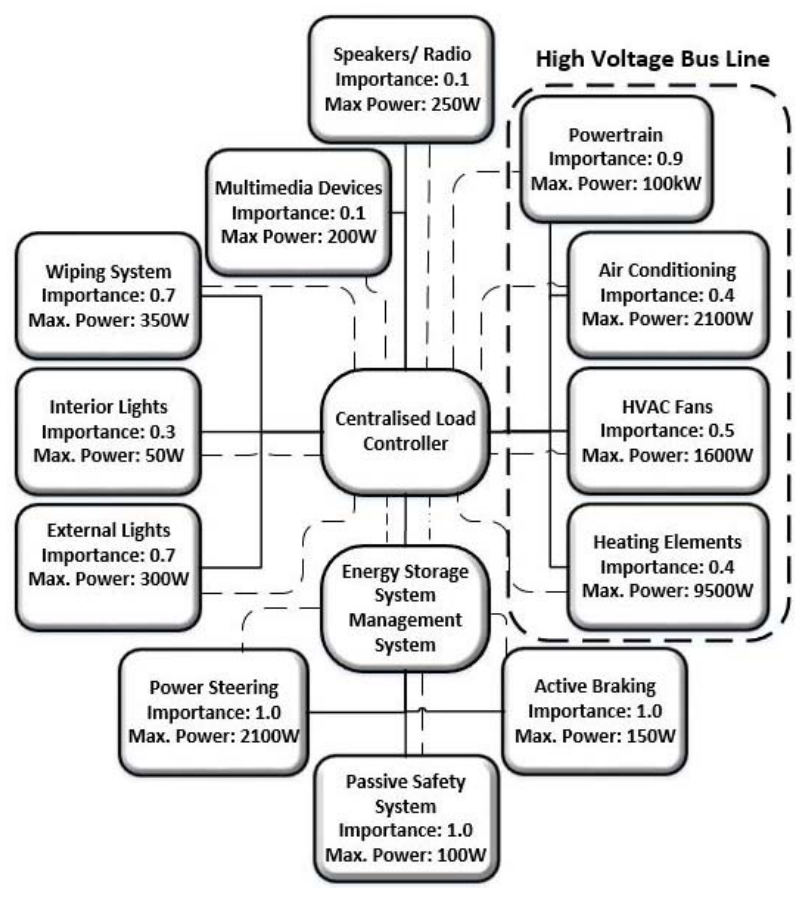

Figure 4. Load Control Architecture

As can be seen on the Fig. 4, there are three types of connection lines. The first type, the solid lines, represents the power flow lines. The second type, dashed line, represents the load request signals. The load request is coming from the individual loads as well as from the centralized load controller to the ESS management system. The last type, dashed-dotted line, represents the signal coming from the ESS to the load controller to make the final decision on the amount of supplied power to the load.

In terms of an overall system design for efficient simulation, ESS should be compatible with the high voltage bus line rather than with the low voltage one. The low voltage bus is supplied via the single direction buck DC-DC converter with the constant 12VDC output voltage. Another power supply for the low voltage bus can be presented as an additional $12 \mathrm{~V}$ battery. Additional battery is required in order to operate with the prompt requests and decrease the waiting time and improve reliability of the system.

In terms of power consumption, the systems were separated into different groups based on their impact on the safety of the vehicle. The importance characteristics were assigned to the components to assess the impact and provide high level of control. The importance have a direct influence on the decision making process for the load controller. The decision making algorithm is presented on Fig. 5.

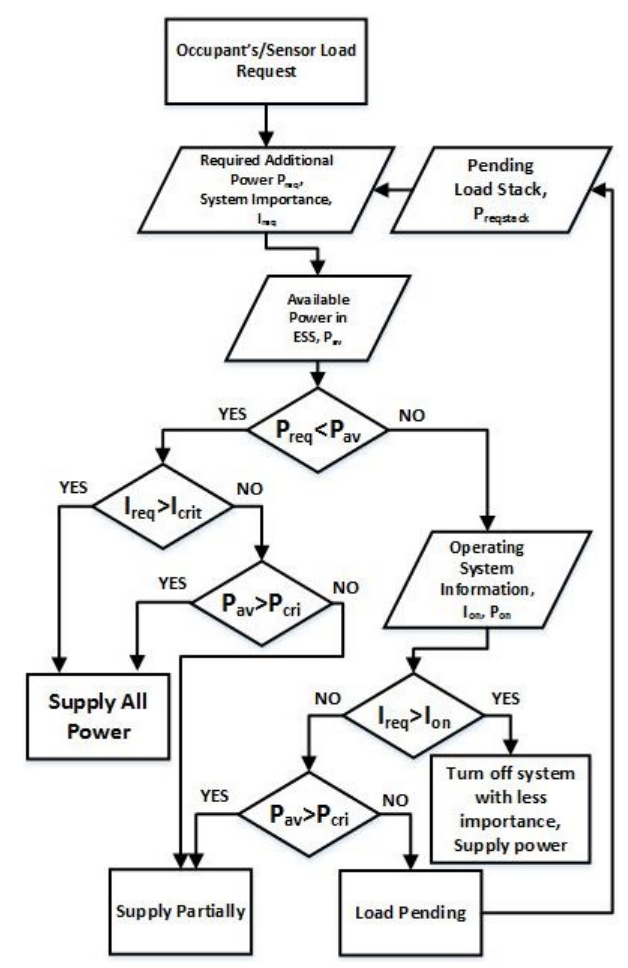

Figure 5. Load Control Algorithm Decision Making Process

Fig. 5 has two main parameters, which defines the decision making process. First parameter is Power and second is Importance, as it was discussed previously. There are three main assessors for these variables. First group of variables is the Request group. This group is defined by default and the information in form of request is coming from the occupants or the sensors for selfoperating systems. Second group of variables is Available values. These values are assigned with the ESS EMS and supplied by the ESS controller. Third group of variables is the predefined croup of critical values. These values have influence on the final decision in terms of the amount of power, which can be supplied to the exact system. Critical values are based on the status of the ESS as the load controller has a direct communication channel with the EMS of ESS. The critical values were predefined on the design stage and they prevent the damage for the components of the ESS while using the most efficient operational region of the various components of the ESS.

\section{ASYNChronous CENTRALISED CONTROLLER}

Conventional synchronous digital circuit design approach deploys a clock system to manage and synchronize the functional operation and timing of the digital circuits. It has been widely adopted by digital circuit designers due to its "easy to understand and manage" design technique. In particular, digital circuits' data paths and control paths must be designed and operated under strict timing constraints dictated by a 
clock signal with specified timing characteristics. In recent years, the design and distribution of such clock system in digital circuits become a significantly challenging task to accomplish due to the relentless integrated-circuit downscaling requirement. As a result, clock-independent or digital design techniques have been proposed as alternative solutions.

Clock-independent circuits are often classified into two types depending on their delay model: bounded delay model (which assumes that the circuit components' delay is bounded and usually requires exhausted timing analysis to ensure operational correctness of the designs), and delay insensitive model (which supposes that the circuit delay is unbounded and the design operation is guaranteed by a specific set of completion conditions of circuit' inputs and outputs). [8] Delay insensitive designs often offer better circuit performance and flexibility compared to their bounded delay counterparts. An extended class of the delay insensitive model is Quasi Delay insensitive (QDI) model in which the delay insensitivity of the digital circuit is fully satisfied with the isochronal fork assumption, where a fan-out signal can reach two or more circuit nodes with insignificant difference in propagation delay. Null Convention Logic (NCL) is a quasi-delay-insensitive digital system design paradigm invented to challenge and eliminates the time dependency problem of synchronous logic. [9] The design approach has steadily developed in recent years, and provides a complete and economically feasible approach to design and implement clock-less digital applications.

NCL circuits are constructed using primitive elements called NCL hysteresis threshold gates THmn illustrated in Fig. 6. Each NCL THmn gate has $\mathrm{n}$ inputs, one output, and $\mathrm{m}$ threshold level. When there is a complete set of $\mathrm{m}$ input DATA (power supply level or Boolean logic 1), the output will be asserted (to power supply level or Boolean logic 1). When all the inputs are NULL (electrical ground level or Boolean logic 0 ), the output will be de-asserted (to ground level or Boolean logic 0 ).

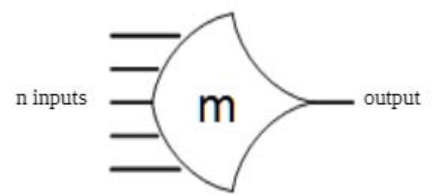

Figure 6. NCL hysteresis threshold gate THmn

The operation and synchronization of NCL circuits, built on those NCL hysteresis threshold gates, is based on successive DATA-NULL wave fronts without the necessity of external synchronization mechanism and hardware (e.g. clock system in synchronous designs) as shown in Fig. 7.

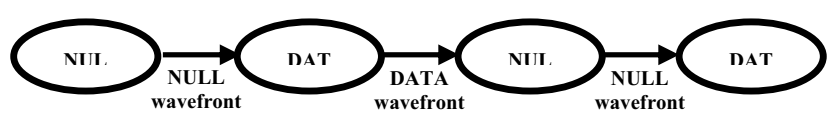

Figure 7: DATA-NULL wave fronts in NCL circuit
Due to the stochastic and unpredictable nature of the power requests in EV systems, the centralized controller must be designed and implemented so that it can asynchronously receive the requests, process them, and provide appropriate response in order to control and manage the power delivery of the energy management system. An asynchronous NCL-based centralized controller, therefore, provides considerably the best possible design choice.

\section{MODELLING OF THE SYSTEM}

\section{A. System Modelling}

Physical model of the hybrid ESS can be implemented using the Simpower library. The main components of the ESS, such as Li-Ion battery, supercapacitor and the fuel cell are available as a part of the existing library.

The control signal for the system is coming from the components, as it can be obtained from the default components. The available models are following the required functions and fully simulate as a real ESS.

The load request, as the input signal, can be simulated using the split approach. In this approach the power request from the powertrain is based on the standard driving cycles, such as New European Driving Cycle (NEDC), and then the random time independent requests for the auxiliary loads can be added in order to simulate the stochastic requests from the occupants and the sensors. At the same time, the developed driving cycles have limited description of the systems operations, so the request for the non-powertrain loads cannot be defined based on the driving cycles.

The simulation of the system energy consumption of the electric systems in an EV can be performed in MatLab Simulink/Simpower environment. The main challenge in terms of the real-world scenario application is to simulate randomized power requests from the components.

The power components can be simulated as the resistive load, as both power busses in EV have DC nature. The intelligent management system was implemented to allocate the assigned amount of energy, which can be supplied in the exact time moment for the each system based on the power request from the other components.

The power regulation was done based on the DC-DC converters, which operates through the high frequency, 20 $\mathrm{kHz}$, insulated-gate bipolar transistors (IGBTs). The controller produces the pulse-width modulated (PWM) signal with variable and adjustable duty cycle, which can be sent to a specifically allocated IGBT switch for each exact load. Due to the DC-DC nature, single IGBT can perform the whole regulation process with the support of the controller, as it is shown on Fig. 8. 


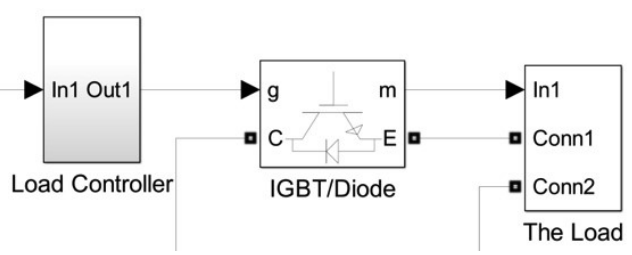

Figure 8. Load Control Model in Matlab

The regulation of the powertrain is different from a normal load control. The bidirectional operational environment of the powerflow requires a four quadrant inverter with variable frequency to control the speed and torque of the motor as well as the direction of the powerflow. For different types of the motors, different inverters can be used.

\section{B. Logic Modelling.}

Physical model of the asynchronous NCL-based centralized controller can be designed and implemented using MATLAB Simscape library. In particular, electronic components required to construct the controller's digital circuit, for example pMOSFET and nMOSFET transistors, are fully supported in the Simscape library. Fig. 9 illustrates a semi-static CMOS NCL hysteresis threshold gate TH23 modelled in MATLAB Simulink environment. Operational characteristics of each MOSFET in the model are tweaked to ensure that the model provides expected functional operation of practical NCL gates designed and implemented in CMOS technology. A complete set of NCL gate library is to be modelled and used to design the centralized controller. Since the operation of NCL-based centralized controller can also be modelled using finite state machine design approach, the functional model of the controller can be done using Stateflow modelling block in MATLAB Simulink environment.

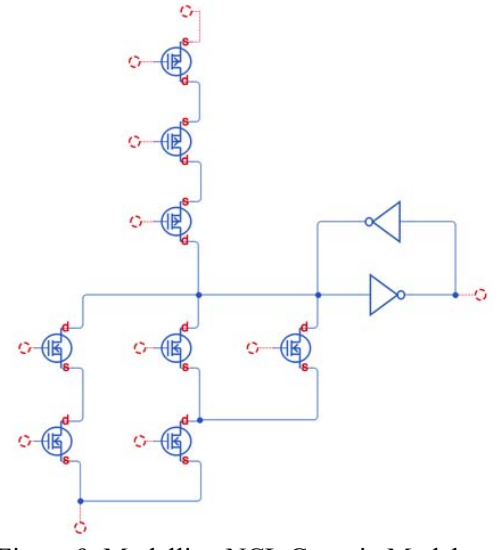

Figure 9. Modelling NCL Gates in Matlab.

\section{CONCLUSION AND WORK IN PROGReSS}

This papers describes the methodology to implement the detailed simulations for centralized load and ESS controller designed using Null Convention Logic in application to the on board system of EV. The Null Convention Logic provides low energy consumption as well as efficient computational time, which has a greater impact on the performance of the controller for a high number of loads with stochastic requests. Centralized architecture provides further benefits in terms of connectivity between the loads and, as a result, higher efficient power consumption with real-time control.

Future work is concentrated on the finite tuning of both the centralized controller with more detailed load requests, and the communication between the load controller and the ESS energy manager system. Another area of the current research is the integration of the solar generation unit in the EMS system and the influence of its input on the overall power consumption.

\section{REFERENCES}

[1] I. Kavalchuk, H. Arisoy, A. T. Oo, and A. Stojcevski, "Challenges of electric power management in hybrid and electric vehicles," in Power Engineering Conference (AUPEC), 2014 Australasian Universities, 2014, pp. 1-7.

[2] K. Vatanparvar, A. Faruque, and M. Abdullah, "Battery lifetimeaware automotive climate control for electric vehicles," in Proceedings of the 52nd Annual Design Automation Conference, 2015, p. 37.

[3] A. Khaligh and Z. Li, "Battery, ultracapacitor, fuel cell, and hybrid energy storage systems for electric, hybrid electric, fuel cell, and plug-in hybrid electric vehicles: State of the art," Vehicular Technology, IEEE Transactions on, vol. 59, pp. 2806-2814, 2010.

[4] J. Moreno, M. E. Ortúzar, and J. W. Dixon, "Energy-management system for a hybrid electric vehicle, using ultracapacitors and neural networks," Industrial Electronics, IEEE Transactions on, vol. 53, pp. 614-623, 2006.

[5] Q. Ren, D. Crolla, and A. Morris, "Effect of transmission design on electric vehicle (EV) performance," in Vehicle Power and Propulsion Conference, 2009. VPPC'09. IEEE, 2009, pp. 12601265.

[6] S. C. Smith and J. Di, "Designing asynchronous circuits using NULL convention logic (NCL)," Synthesis Lectures on Digital Circuits and Systems, vol. 4, pp. 1-96, 2009.

[7] G. Kowalczyk, M. Dielacher, M. Flatscher, J. Prainsack, H. Unterassinger, J. Schweighofer, et al., "Asynchronous logic application in a power management unit for ultra low power wireless sensor nodes," in AFRICON, 2011, 2011, pp. 1-6.

[8] S. Hauck, "Asynchronous design methodologies: An overview," Proceedings of the IEEE, vol. 83, pp. 69-93, 1995.

[9] K. M. Fant, Logically determined design: clockless system design with NULL convention logic: John Wiley \& Sons, 2005. 